EP-23

\title{
Modified liver hanging maneuvers to facilitate various difficult types of hepatectomy: A single center experience
}

\author{
Giang NGUYEN*, Anh PHAM, Trong NGUYEN, Hung NGUYEN, Ha NGHIEM, Duc PHAM
}

Hepatobiliary and Pancreatic Surgery Department, Vietnam National Cancer Hospital, Hanoi, Vietnam

Introduction: The liver hanging maneuver (LHM) is a useful technique enabling a safe anterior approach. Since the first time described by Belghiti, it has been modified many times by different surgeons to be more effective. Thus, we aim to evaluate the short term results of using some modified liver hanging maneuvers (mLHM) for difficult types of hepatectomy.

Methods: From February 1 to September 30, 2020, we perform 3 modified techniques of LHM for 4 patients with liver's tumors. mLHM 1: A tape was placed upon the fossa ductus venosi, its cranial tip was passed to the right behind the common trunk of left hepatic vein and middle heptic vein, and its caudal tip passed behind the left Glissonean pedicle to the hepatic hilum. mLHM 2: After transect the caudal part of S1R about $2 \mathrm{~cm}$, a tape was passed through the hepatic hilum and the right hepatic vein - middle hepatic vein pocket along the right border of the paracaval portion. mLHM 3: Instead of a tape, we used 2 fingers to create the tunnel and push the liver forward.

Results: Among 4 patients, there were 2 right hepatectomy and caudate lobectomy, 1 left hepatectomy and caudate lobectomy, 1 right anterior sectionectomy with reconstruction right hepatic vein for huge tumor. The modified LHMs were performed successfully in all patients, provide adequate cut planes without complications.

Conclusions: The LHM is a safe and effective technique which can be modified to adapt with many types of hepatectomy.

Key Words: Modified liver hanging maneveur; Caudate lobectomy; Huge tumor 